\title{
MELTING HEAT TRANSFER IN A NANOFLUID FLOW PAST A PERMEABLE CONTINUOUS MOVNG SURFACE
}

\author{
Rama Subba Reddy Gorla $^{a}$, Ali Chamkha ${ }^{b}$, Abdulkareem Aloraier $^{b}$
}

a Department of Mechanical Engineering, Cleveland State University, Cleveland, Ohio, USA. Email: r.gorla@csuohio.edu

${ }^{\mathrm{b}}$ Public Authority for Applied Education and Training, Shuweikh, Kuwait

\begin{abstract}
:
A boundary layer analysis is presented for the warm, laminar nanoliquid flow to a melting surface moving parallel to a uniform free stream. The resulting system of non-linear ordinary differential equations is solved numerically using Runge-Kutta method with shooting techniques. Numerical results are obtained for the velocity, temperature and concentration distributions, as well as the friction factor, local Nusselt number and local Sherwood number for several values of the parameters, namely the velocity ratio parameter, melting parameter and nanofluid parameters. The obtained results are presented graphically and in tabular form and the physical aspects of the problem are discussed.
\end{abstract}

Keywords: Suction/injection, moving surface, nanofluid, boundary layer, shooting technique.

\section{NOMENCLATURE}

$\mathrm{C}$

$\mathrm{D}_{\mathrm{B}} \quad$ Brownian diffusion coefficient

$\mathrm{D}_{\mathrm{T}} \quad$ thermophoretic diffusion coefficent

f reduced stream function

g gravitational acceleration

$\mathrm{k}_{\mathrm{m}}$ effective thermal conductivity of the porous material

$\mathrm{K}$ permeability of porous medium

Le Lewis number

M melting parameter

$\mathrm{N}_{\mathrm{b}} \quad$ Brownian motion parameters

$\mathrm{N}_{\mathrm{t}}$ thermophoresis parameters

$\mathrm{Nu} \quad$ Nusselt number

$\mathrm{p}$ pressure

q wall heat flux

Re Reynolds number

$\mathrm{T}$ temperature

$\mathrm{T}_{\mathrm{m}} \quad$ melting surface temperature

$\mathrm{T}_{0} \quad$ solid surface temperature

$\mathrm{T}_{\infty} \quad$ ambient temperature

$\mathrm{u}_{\infty} \quad$ freestream velocity

$\mathrm{u}, \mathrm{v} \quad$ Darcy velocity components

$(\mathrm{x}, \mathrm{y}) \quad$ Cartesian coordinates
Greek Symbols:

$\alpha_{m} \quad$ thermal diffusivity of porous medium

$\eta \quad$ dimensionless distance

$\theta$ dimensionless temperature

$\mu \quad$ viscosity of fluid

$\rho_{\mathrm{f}} \quad$ fluid density

$\rho_{p} \quad$ nano-particle mass density

$(\rho \mathrm{C})_{\mathrm{f}} \quad$ heat capacity of the fluid

$(\rho \mathrm{C})_{\mathrm{m}} \quad$ effective heat capacity of porous medium

$(\rho C)_{p}$ effective heat capacity of nano-particle material

$\tau \quad$ ratio between the effective heat capacity of the nano particle material and that of the fluid

c nano-particle volume fraction

$\mathrm{c}_{\mathrm{w}}$ nano-particle volume fraction at the wall of the plate

$\mathrm{c}_{\infty}$ ambient nano-particle volume fraction

$\psi \quad$ stream function

Subscripts

B Blasius problem

S Sakiadis problem

w Refers to condition at wall

$\infty \quad$ Refers to condition far from the wall 


\section{Introduction}

The study of convective heat transfer in nanofluids is gaining a lot of attention. The nanofluids have many applications in the industries since materials of nanometer size have unique physical and chemical properties. Nanofluids are solid-liquid composite materials consisting of solid nanoparticles or nanofibers with sizes typically of 1-100 nm suspended in liquid. Nanofluids have attracted great interest recently because of reports of greatly enhanced thermal properties. For example, a small amount $(<1 \%$ volume fraction) of $\mathrm{Cu}$ nanoparticles or carbon nanotubes dispersed in ethylene glycol or oil is reported to increase the inherently poor thermal conductivity of the liquid by $40 \%$ and $150 \%$ respectively by Eastman et al. (2001) and Choi et al. (2001). Conventional particle-liquid suspensions require high concentration $(>10 \%)$ of particles to achieve such enhancement. However, problems of rheology and stability are amplified at high concentration, precluding the widespread use of conventional slurries as heat transfer fluids. In some cases, the observed enhancement in thermal conductivity of nanofluids is orders of magnitude larger than predicted by well-established theories. Other perplexing results in this rapidly evolving field include a surprisingly strong temperature dependence of the thermal conductivity as reported by Patel et al. (2003) and a three-fold higher critical heat flux compared with the base fluids as reported by You et al. (2003). These enhanced thermal properties are not merely of academic interest. If confirmed and found consistent, they would make nanofluids promising for application in thermal management. Furthermore, suspensions of metal nanoparticles are also being developed for other purposes, such as medical applications including cancer therapy. The interdisciplinary nature of nanofluid research presents a great opportunity for exploration and discovery at the frontiers of nanotechnology.

The characteristics of flow and heat transfer of a viscous and incompressible fluid over flexed or continuously moving flat surfaces in a moving or a quiescent fluid are well understood. These flows occur in many manufacturing processes in modern industry, such as hot rolling, hot extrusion, wire drawing and continuous casting. For example, in many metallurgical processes such as drawing of continuous filaments through quiescent fluids and annealing and tinning of copper wires, the properties of the end product depends greatly on the rare of cooling involved in these processes. Sakiadis (1961) was the first one to analyze the boundary layer flow on continuous surfaces. Crane (1972) obtained an exact solution the boundary layer flow of Newtonian fluid caused by the stretching of an elastic sheet moving in its own plane linearly. Tsou et al. (1967) extended the research to the heat transfer phenomenon of the boundary layer flow on a continuous moving surface. Schowalter (1960) applied the boundary layer theory into power law pseudoplastic fluids and developed two and three dimensional boundary layer equations of the momentum transfer. Acrivos (1960) analyzed the momentum and heat transfer of non-Newtonian fluid past arbitrary external surfaces. Howell et al. (1997) and Rao et al. (1999) investigated momentum and heat transfer phenomena on a continuous moving surface in power law fluids. Magyari and Keller (1999) have studied the thermal boundary layer of moving surfaces. Wang (1989) studied free convection from a vertical stretching surface. Gorla and Sidawi (1994) studied the characteristics of flow and heat transfer from a continuous surface with suction and blowing.

A survey of convective heat transfer in nanofluids has been presented by Buongiorno and $\mathrm{Hu}(2005)$ as well as Kakac and Pramuanjaroenkij (2009). Numerous models have been proposed to study convective flows of nanofluids reported by Tiwari et al. (2007), Wang and Wei (2009), Nield and Kuznetsov (2009) and Gorla et al. (2011).

Phase change heat transfer finds applications in magma solidification, permafrost melting, preparation of semiconductor materials etc. The analogy between melting and diffusion mass transfer or transpiration cooling was noted by Huang and Shih (1975). Pedroso and Domoto (1973) developed a method for calculating melting rates based on the diffusion/melting analogy. We present here a similarity analysis for the problem of steady boundary-layer flow and heat transfer from a warm, laminar liquid flow to a melting surface moving parallel to a constant free stream of a nanofluid. It is assumed that the melting of the plate takes place at a steady state. The development of the velocity, temperature and concentration distributions have been illustrated for several values of nanofluid parameters, Prandtl number, Lewis number, velocity ratio and suction/injection parameters.

\section{Analysis}

Consider a flat surface melting at a steady rate into a constant property warm liquid of the same material. The surface is assumed to be moving at a constant velocity $u_{\mathrm{w}}$ in a parallel direction to a free stream of a nanofluid of uniform velocity $u_{\infty}$. The flow model and coordinate system are shown in Figure 1. Either the surface velocity or the free-stream velocity may be zero but not both at the same time. The physical properties of the fluid are 
assumed to be constant. It is assumed that the temperature of the melting surface is $T_{m}$ while the free stream temperature $T_{\infty}>T_{m}$. Under such condition, the governing equations of the steady, laminar boundary-layer flow on the moving surface are given by:

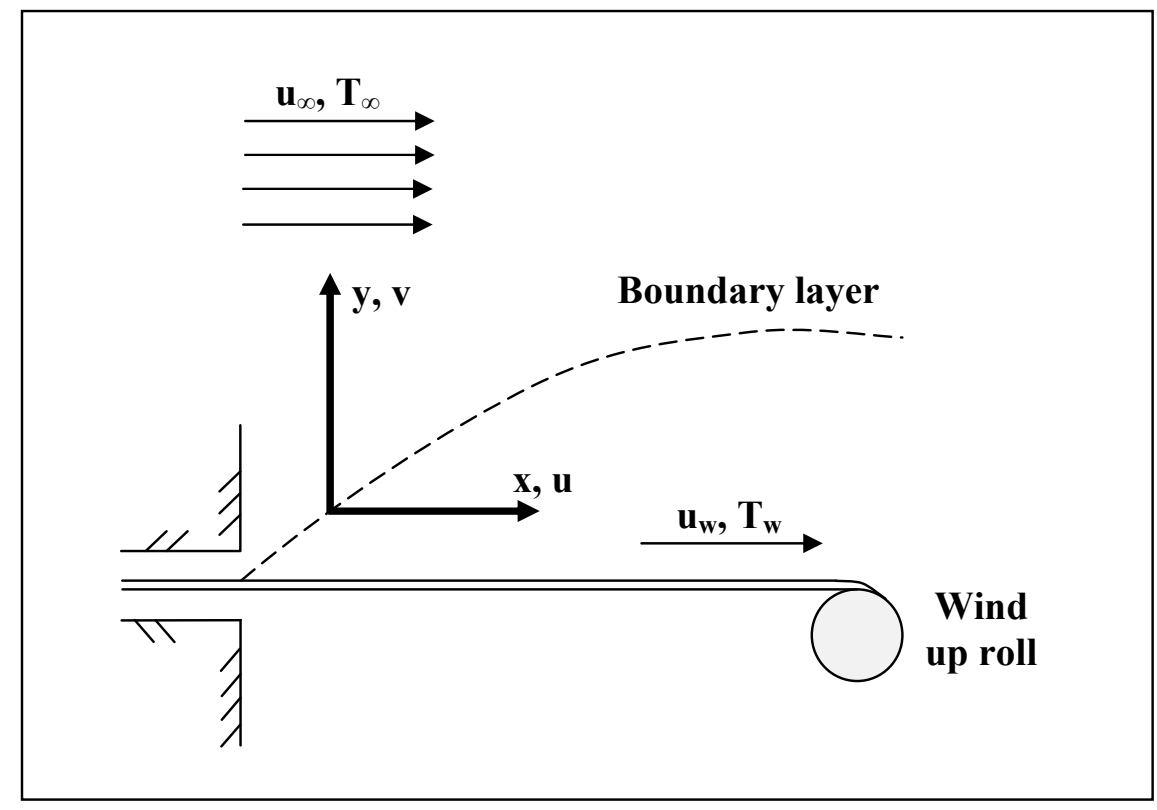

Figure 1: Flow Model and Coordinate System

$\frac{\partial u}{\partial x}+\frac{\partial v}{\partial y}=0$

$u \frac{\partial u}{\partial x}+v \frac{\partial u}{\partial y}=-\frac{1}{\rho_{\mathrm{f}}} \frac{\partial p}{\partial x}+v\left(\frac{\partial^{2} u}{\partial x^{2}}+\frac{\partial^{2} u}{\partial y^{2}}\right)$

$u \frac{\partial v}{\partial x}+v \frac{\partial v}{\partial y}=-\frac{1}{\rho_{\mathrm{f}}} \frac{\partial p}{\partial y}+v\left(\frac{\partial^{2} v}{\partial x^{2}}+\frac{\partial^{2} v}{\partial y^{2}}\right)$

$u \frac{\partial T}{\partial x}+v \frac{\partial T}{\partial y}=\alpha\left(\frac{\partial^{2} T}{\partial x^{2}}+\frac{\partial^{2} T}{\partial y^{2}}\right)+\tau\left\{\mathrm{D}_{\mathrm{B}}\left(\frac{\partial \mathrm{c}}{\partial \mathrm{x}} \frac{\partial \mathrm{T}}{\partial \mathrm{x}}+\frac{\partial \mathrm{c}}{\partial \mathrm{y}} \frac{\partial \mathrm{T}}{\partial \mathrm{y}}\right)+\left(\frac{\mathrm{D}_{\mathrm{T}}}{\mathrm{T}_{\infty}}\right)\left[\left(\frac{\partial \mathrm{T}}{\partial \mathrm{x}}\right)^{2}+\left(\frac{\partial \mathrm{T}}{\partial \mathrm{y}}\right)^{2}\right]\right\}$

$u \frac{\partial c}{\partial x}+v \frac{\partial c}{\partial y}=\mathrm{D}_{\mathrm{B}}\left(\frac{\partial^{2} c}{\partial x^{2}}+\frac{\partial^{2} T}{\partial y^{2}}\right)+\left(\frac{\mathrm{D}_{\mathrm{T}}}{\mathrm{T}_{\infty}}\right)\left(\frac{\partial^{2} T}{\partial x^{2}}+\frac{\partial^{2} T}{\partial y^{2}}\right)$

The boundary conditions are given by,

$y=0: u=u_{\mathrm{w}}, v=v_{\mathrm{w}}(\mathrm{x}), k \frac{\partial T}{\partial y}=\rho_{f}\left[\lambda+C_{S}\left(T_{m}-T_{0}\right)\right] v_{w}, \mathrm{c}=\mathrm{c}_{\mathrm{w}}$

$y \rightarrow \infty: u=\mathrm{u}_{\infty}, T=T_{\infty}, \mathrm{c}=\mathrm{c}_{\infty}$

The boundary condition of $u=u_{\mathrm{w}}$ in (6) represents the case of a plane surface moving in parallel to the free stream.

To analyze the effect of both the moving and the free stream on the boundary-layer flow, we propose a new similarly coordinate and a dimensionless stream function

$\eta=\frac{y}{x}\left(R e_{w}+R e_{\infty}\right)^{\frac{1}{2}}, \quad \quad f=\frac{\psi}{v\left(R e_{w}+R e_{\infty}\right)^{\frac{1}{2}}}$

which are the combinations of the traditional ones:

$\eta_{B}=\frac{y}{x} R e_{\infty}^{\frac{1}{2}}, \quad \quad f_{B}=\frac{\psi}{v R e_{\infty}^{\frac{1}{2}}}$

for the Blasius problem (stationary wall and uniform freestream velocity) and

$\eta_{S}=\frac{y}{x} R e_{w}^{\frac{1}{2}}, \quad f_{S}=\frac{\psi}{v R e_{w}^{\frac{1}{2}}}$

from the Sakiadis (uniformly moving wall with stagnant freestream) problem. 
The Reynolds numbers are defined as:

$R e_{w}=\frac{u_{w} x}{v}, \quad R e_{\infty}=\frac{u_{\infty} x}{v}$

A velocity ratio parameter $\gamma$ is defined as

$\gamma=\frac{\mathrm{u}_{\mathrm{w}}}{\left(\mathrm{u}_{\mathrm{w}}+\mathrm{u}_{\infty}\right)}=\left(1+\frac{\mathrm{u}_{\infty}}{\mathrm{u}_{\mathrm{w}}}\right)^{-1}=\left(1+\frac{\mathrm{Re}_{\infty}}{\mathrm{Re}_{\mathrm{w}}}\right)^{-1}$,

Note that form the Blasius problem, $\mathrm{u}_{\mathrm{w}}=0$ therefore $\gamma=0$. On the other hand, for the Sakiadis problem, $\mathrm{u}_{\infty}=0$ and thus $\gamma=1$. In addition, we also define dimensionless temperature and concentration function as

$\theta=\frac{T-T_{\infty}}{T_{m}-T_{\infty}}$

$\phi=\frac{c-c_{\infty}}{c_{w}-c_{\infty}}$

Using the transformation variables defined in Equations (7) - (13), the governing transformed equations may be written as

$f^{\prime \prime \prime}+\frac{f f^{\prime \prime}}{2}=0$

$\frac{\theta^{\prime \prime}}{P r}+\frac{f \theta^{\prime}}{2}+N_{b} \phi^{\prime} \theta^{\prime}+N_{t}\left(\theta^{\prime}\right)^{2}=0$

$\phi^{\prime \prime}+\frac{\text { Le.f } \phi^{\prime}}{2}+\frac{N_{t}}{N_{b}} \theta^{\prime \prime}=0$

The transformed boundary conditions are given by

Pr. $f(0)+2 M \theta^{\prime}(0)=0, \quad f^{\prime}(0)=\gamma, \quad \theta(0)=1, \quad \phi(0)=1$

$f^{\prime}(\infty)=1-\gamma, \quad \theta(\infty)=0, \quad \emptyset(\infty)=0$

where prime denote differentiation with respect to $\eta$ and the four parameters are defined by

$\operatorname{Pr}=\frac{v}{\alpha}, \quad L e=\frac{v}{D_{B}}, M=\frac{C_{p}\left(T_{\infty}-T_{m}\right)}{\lambda+C_{S}\left(T_{m}-T_{0}\right)}$

$N_{b}=\frac{(\rho c)_{p} D_{B}\left(\phi_{w}-\phi_{\infty}\right)}{(\rho c)_{f} v}$,

$N_{t}=\frac{(\rho c)_{p} D_{T}\left(T_{m}-T_{\infty}\right)}{(\rho c)_{f} T_{\infty} v}$

Here, $\operatorname{Pr}, L e, N_{b}$ and $N_{t}$ denote the Prandtl number, the Lewis number, the Brownian motion parameter and the thermophoresis parameter respectively. It is important to note that this boundary value problem reduces to the classical problem of flow and heat and mass transfer due to a stretching surface in a viscous fluid when $\mathrm{N}_{b}$ and $\mathrm{N}_{\mathrm{t}}$ are zero.

The quantities of practical interest, in this study, are the Nusselt number $N u$ and the Sherwood number $S h$ which are defined as:

Friction Factor

$$
\begin{aligned}
& C_{\infty}=\frac{\tau_{w}}{\left(\frac{\rho u_{\infty}{ }^{2}}{2}\right)}=2 R e_{\infty}^{-\frac{1}{2}}(1-\gamma)^{-\frac{3}{4}}\left|f^{\prime \prime}(0)\right| \\
& C_{w}=\frac{\tau_{w}}{\left(\frac{\rho u_{w}{ }^{2}}{2}\right)}=2 R e_{w}^{-\frac{1}{2}} \gamma^{-\frac{3}{4}}\left|f^{\prime \prime}(0)\right|
\end{aligned}
$$

The local heat transfer rate (Local Nusselt number) is given by

$N u_{x}=\frac{q_{w} x}{R\left(T_{w}-T_{\infty}\right)}=-\frac{R \theta^{\prime}(0)}{R} \frac{\left(u_{w}-u_{\infty}\right)^{\frac{1}{2}}}{\vartheta^{-\frac{1}{2}} x^{-\frac{1}{2}}} x=-\left(R e_{w}-R e_{\infty}\right)^{\frac{1}{2}} \theta^{\prime}(0)$

Similarly the local Sherwood number is given by

$S h_{x}=\frac{q_{m} x}{D_{B}\left(C_{w}-c_{\infty}\right)}=-\left(R e_{w}-R e_{\infty}\right)^{\frac{1}{2}} \phi^{\prime}(0)$

where $\mathrm{q}_{\mathrm{w}}$ and $\mathrm{q}_{\mathrm{m}}$ are wall heat and mass flux rates, respectively.

\section{Results and discussions}

The nonlinear ordinary differential equations (14)-(16), satisfying the boundary conditions (17) were integrated numerically by using the fourth-order Runge-Kutta scheme along with the shooting method for several values of the governing parameters, namely, Prandtl number (Pr), Lewis number (Le), Brownian motion parameter $\left(\mathrm{N}_{\mathrm{b}}\right)$ and thermophoresis parameter $\left(\mathrm{N}_{\mathrm{t}}\right)$. It may be noted that $\operatorname{Pr}=0.007$ corresponds to liquid metal Gallium and $\mathrm{Pr}$ $=0.025$ to liquid metal germanium. In order to assess the accuracy of the present results, we obtained results for the reduced Nusselt number $-\theta^{\prime}(0)$ by ignoring the effects of $\mathrm{M}, \mathrm{N}_{\mathrm{b}}$ and $\mathrm{N}_{\mathrm{t}}$. These results are shown in Table 1 . A comparison of our results with literature values indicates excellent agreement and therefore our results are highly accurate.

Melting heat transfer in a nanofluid flow past a permeable continuous moving surface 
Table 1: Comparison of results for $-\theta^{\prime}(0)$ for the Sakiadis problem $\quad\left(M=N_{t}=N_{b}=0 ; \gamma=1\right)$

\begin{tabular}{|l|l|l|c|}
\hline $\operatorname{Pr}$ & Present results & Wang [14] & Gorla and Sidawi [15] \\
\hline 0.07 & 0.0656 & 0.0656 & 0.0656 \\
\hline 0.20 & 0.1691 & 0.1691 & 0.1691 \\
\hline 0.70 & 0.4539 & 0.4539 & 0.4539 \\
\hline 7.00 & 1.8907 & 1.8954 & 1.8905 \\
\hline 20.00 & 3.3539 & 3.3539 & 3.3539 \\
\hline 70.00 & 6.4622 & 6.4622 & 6.4622 \\
\hline
\end{tabular}

Table 2: Effects of $M$ on $f^{\prime \prime}(0),-\theta^{\prime}(0)$ and $-\varphi^{\prime}(0)$ for $\mathrm{N}_{\mathrm{b}}=0.3, \mathrm{~N}_{\mathrm{t}}=0.1, \mathrm{Le}=10, \operatorname{Pr}=0.007$ and $\gamma=0.1$

\begin{tabular}{|l|l|l|l|}
\hline $\mathrm{M}$ & $\mathrm{f}^{\prime}(0)$ & $-\theta^{\prime}(0)$ & $-\varphi^{\prime}(0)$ \\
\hline 0 & $2.783765 \mathrm{E}-01$ & $4.241628 \mathrm{E}-02$ & $8.482305 \mathrm{E}-01$ \\
\hline 0.5 & $3.170545 \mathrm{E}-01$ & $4.246388 \mathrm{E}-02$ & 1.310642 \\
\hline 1.0 & $3.547819 \mathrm{E}-01$ & $4.313251 \mathrm{E}-02$ & 1.811196 \\
\hline 2.0 & $4.280522 \mathrm{E}-01$ & $4.374699 \mathrm{E}-02$ & 2.846745 \\
\hline 3.0 & $4.985808 \mathrm{E}-01$ & $4.482413 \mathrm{E}-02$ & 3.868566 \\
\hline
\end{tabular}

Tables 2 displays the resulting values of velocity gradient $f^{\prime \prime}(0)$, the sheet surface heat transfer rate $-\theta^{\prime}(0)$ and the mass transfer rate $-\phi^{\prime}(0)$, which are proportional to the friction factor, Nusselt number and Sherwood number respectively, for several values of the melting parameter, $M$. The results indicate that as $\mathrm{M}$ increases, the friction factor, heat transfer rate and mass transfer rate increase. Thus, increasing the melting strength increases the heat and mass transfer rates at the solid/fluid interface. Melting provides a blowing condition at the moving surface. The results in Table 3 show that as the velocity ratio $\gamma$ increases, the heat transfer rates decrease and mass transfer rates increase. The results in Table 4 show that as the Lewis number, Le increases, the heat transfer and mass transfer rates increase. The results in Table 5 show that as the thermophoresis parameter Nt increases, the heat transfer rates decrease and mass transfer rates increase. The results in Table 6 show that as the Brownian motion parameter $\left(\mathrm{N}_{\mathrm{b}}\right)$ increases, the heat transfer rates decrease and mass transfer rates increase. As the Prandtl number Pr increases, the friction factor increases and in consequence increases the heat transfer rate at the surface.

Table 3: Effects of $\gamma$ on $f^{\prime \prime}(0),-\theta^{\prime}(0)$ and $-\varphi^{\prime}(0)$ for $M=1.0, N_{b}=0.3, N_{t}=0.1, L e=10$ and $\operatorname{Pr}=0.007$

\begin{tabular}{|l|l|l|l|}
\hline$\gamma$ & $f^{\prime}(0)$ & $-\theta^{\prime}(0)$ & $-\varphi^{\prime}(0)$ \\
\hline 0 & $4.020455 \mathrm{E}-01$ & $4.457481 \mathrm{E}-02$ & 1.393198 \\
\hline 0.25 & $2.616510 \mathrm{E}-01$ & $4.082558 \mathrm{E}-02$ & 2.674506 \\
\hline 0.5 & $-8.41530 \mathrm{E}-05$ & $3.644821 \mathrm{E}-02$ & 5.225882 \\
\hline 0.75 & $-5.92690 \mathrm{E}-01$ & $3.191911 \mathrm{E}-02$ & 10.236470 \\
\hline 1.0 & -1.987978 & $2.772456 \mathrm{E}-02$ & 18.883270 \\
\hline
\end{tabular}

Table 4: Effects of $\mathrm{L}$ on $\mathrm{f}^{\prime \prime}(0),-\theta^{\prime}(0)$ and $-\varphi^{\prime}(0)$ for $\mathrm{M}=1.0, \mathrm{~N}_{\mathrm{b}}=0.3, \mathrm{~N}_{\mathrm{t}}=0.1, \operatorname{Pr}=0.007$ and $\gamma=0.1$

\begin{tabular}{|l|l|l|l|}
\hline Le & $f^{\prime}(0)$ & $-\theta^{\prime}(0)$ & $-\varphi^{\prime}(0)$ \\
\hline 1 & $3.547975 \mathrm{E}-01$ & $4.321312 \mathrm{E}-02$ & $4.426402 \mathrm{E}-01$ \\
\hline 10 & $3.547819 \mathrm{E}-01$ & $4.313251 \mathrm{E}-02$ & 1.811196 \\
\hline $10^{2}$ & $3.547536 \mathrm{E}-01$ & $4.342895 \mathrm{E}-02$ & 13.631110 \\
\hline $10^{3}$ & $3.547498 \mathrm{E}-01$ & $4.330121 \mathrm{E}-02$ & 132.718400 \\
\hline
\end{tabular}


Table 5: Effects of $\mathrm{N}_{\mathrm{t}}$ on $\mathrm{f}^{\prime \prime}(0),-\theta^{\prime}(0)$ and $-\varphi^{\prime}(0)$ for $\mathrm{M}=0.5, \mathrm{~N}_{\mathrm{b}}=0.3, \mathrm{Le}=10, \mathrm{Pr}=1.0$ and $\gamma=0.1$

\begin{tabular}{|l|l|l|l|}
\hline $\mathrm{N}_{\mathrm{t}}$ & $\mathrm{f}^{\prime}(0)$ & $-\theta^{\prime}(0)$ & $-\varphi^{\prime}(0)$ \\
\hline 0.1 & $1.501518 \mathrm{E}-01$ & $2.820203 \mathrm{E}-01$ & 1.063284 \\
\hline 0.2 & $1.501518 \mathrm{E}-01$ & $2.690583 \mathrm{E}-01$ & 1.063858 \\
\hline 0.3 & $1.501518 \mathrm{E}-01$ & $2.571833 \mathrm{E}-01$ & 1.068012 \\
\hline 0.4 & $1.501518 \mathrm{E}-01$ & $2.457135 \mathrm{E}-01$ & 1.075496 \\
\hline 0.5 & $1.501518 \mathrm{E}-01$ & $2.348450 \mathrm{E}-01$ & 1.086077 \\
\hline
\end{tabular}

Table 6: Effects of $\mathrm{N}_{\mathrm{b}}$ on $\mathrm{f}^{\prime \prime}(0),-\theta^{\prime}(0)$ and $-\varphi^{\prime}(0)$ for $\mathrm{M}=0.5, \mathrm{~N}_{\mathrm{t}}=0.3, \mathrm{Le}=10, \operatorname{Pr}=1.0$ and $\gamma=0.1$

\begin{tabular}{|l|l|l|l|}
\hline $\mathrm{N}_{\mathrm{b}}$ & $\mathrm{f}^{\prime}(0)$ & $-\theta^{\prime}(0)$ & $-\varphi^{\prime}(0)$ \\
\hline 0.1 & $1.501518 \mathrm{E}-01$ & $3.021600 \mathrm{E}-01$ & $9.746230 \mathrm{E}-01$ \\
\hline 0.2 & $1.501518 \mathrm{E}-01$ & $2.791630 \mathrm{E}-01$ & 1.045009 \\
\hline 0.3 & $1.501518 \mathrm{E}-01$ & $2.571833 \mathrm{E}-01$ & 1.068012 \\
\hline 0.4 & $1.501518 \mathrm{E}-01$ & $2.369526 \mathrm{E}-01$ & 1.078316 \\
\hline 0.5 & $1.501518 \mathrm{E}-01$ & $2.185187 \mathrm{E}-01$ & 1.083437 \\
\hline
\end{tabular}

Figures 2-4 display results for the variation of velocity, temperature and concentration within the boundary layer. As the melting parameter $\mathrm{M}$ increases, the velocity increases whereas the temperature and concentration decrease. Figure 5 shows the velocity distribution within the boundary layer for several values of the velocity ratio parameter $\gamma$. A value of zero for the velocity parameter $\gamma$ describes the Blasius problem whereas $\gamma=1$ describes the Sakiadis problem. Figure 6 and 7 show the temperature and concentration distribution within the boundary layer for several values of the velocity ratio parameter $\gamma$. As $\gamma$ increases, the temperature increases while the concentration values decrease. Figures 8 and 9 show the temperature and concentration distribution as the Lewis number increases. As Le increases, we observe that the concentration decreases and the concentration boundary layer thickness decreases. This in turn increases the surface mass transfer rates as Le increases.

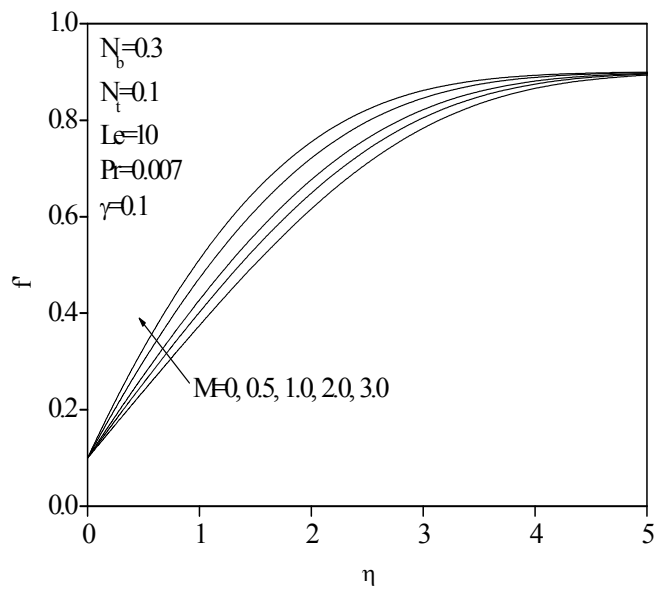

Figure 2: Effects of $\mathrm{M}$ on velocity profiles

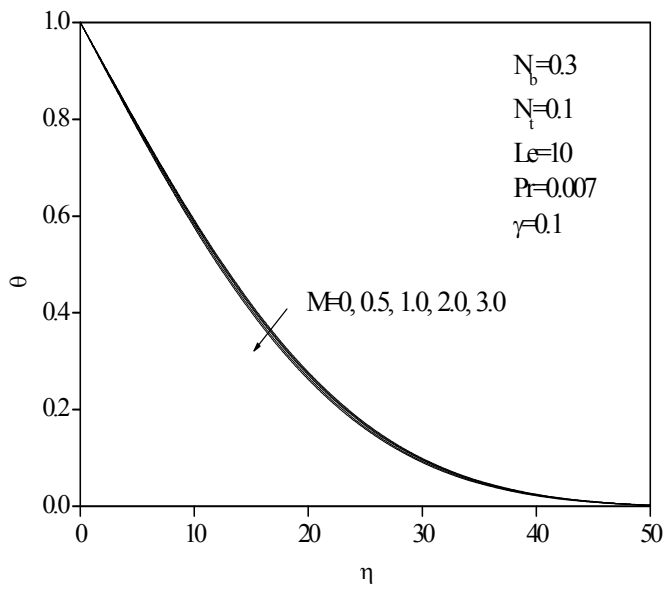

Figure 3: Effects of $\mathrm{M}$ on temperature profiles 
R. S. R.. Gorla , A. Chamkha, A. Aloraier / Journal of Naval Architecture and Marine Engineering 2(2011) 83-92

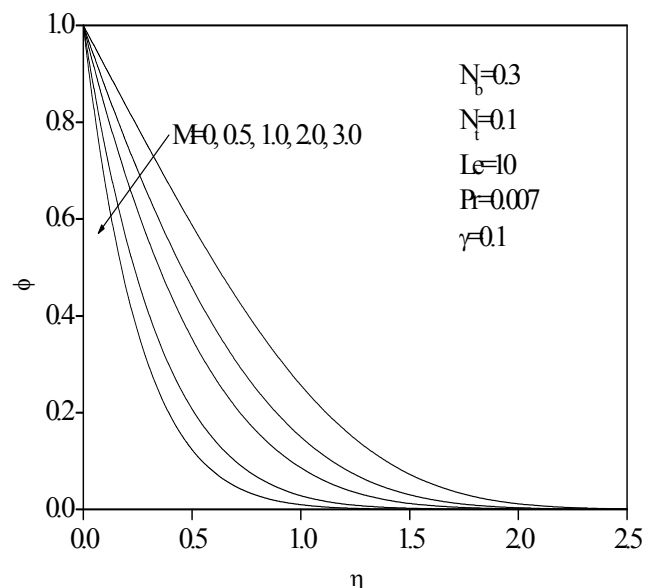

Figure 4: Effects of $\mathrm{M}$ on solid volume fraction profiles

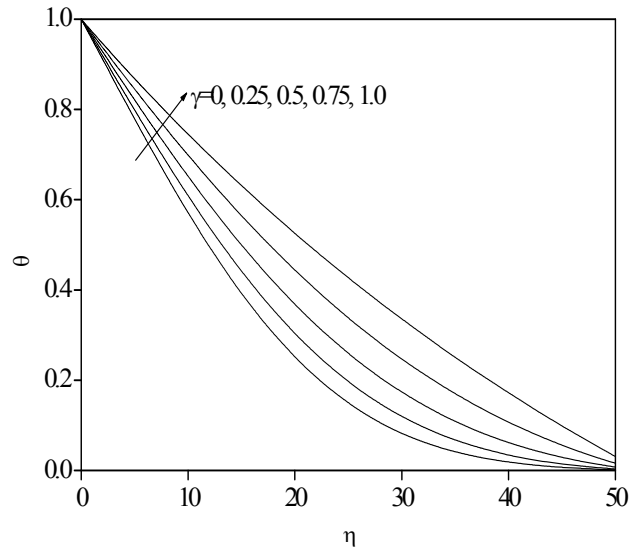

Figure 6: Effects of $\gamma$ on temperature profiles

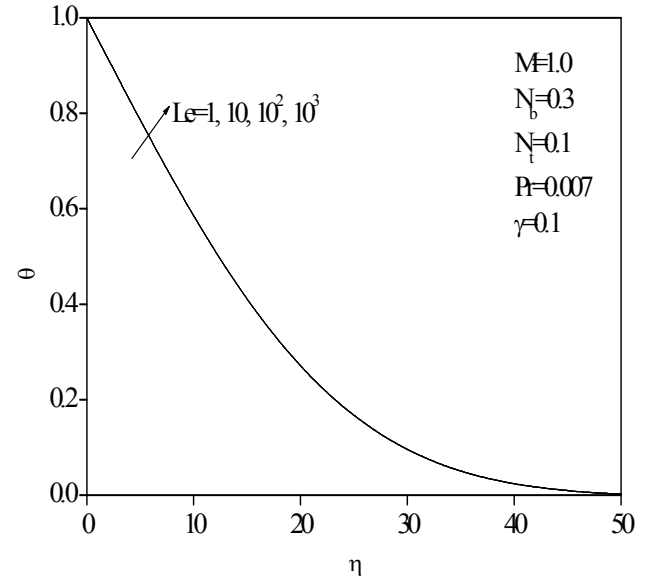

Figure 8: Effects of Le on temperature profiles

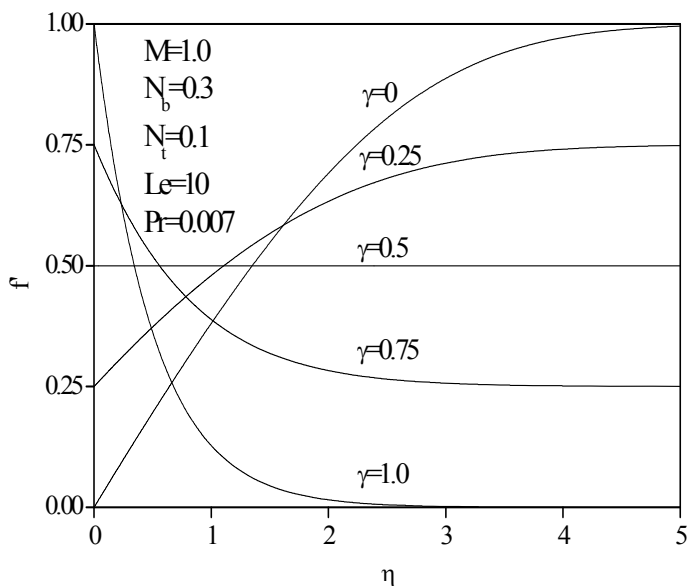

Figure 5: Effects of $\gamma$ on velocity profiles

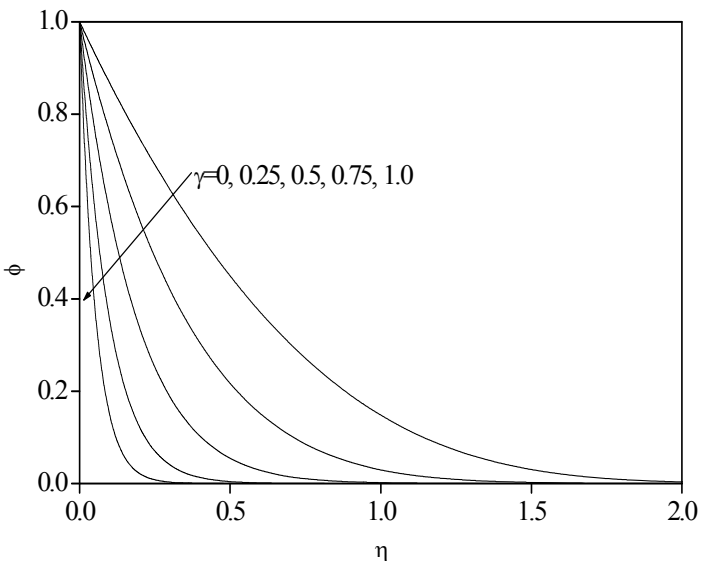

Figure 7: Effects of $\gamma$ on solid volume fraction profiles

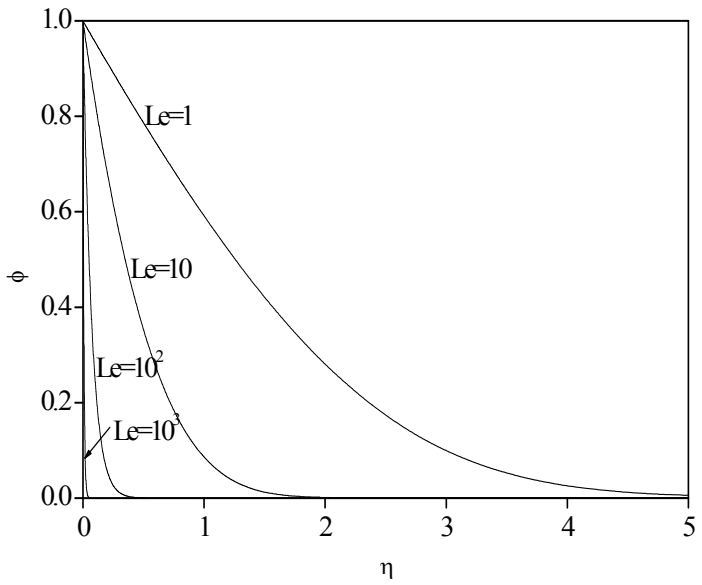

Figure 9: Effects of Le on solid volume fraction profiles 
Figures 10 and 11 show the variation of temperature and concentration as $N_{t}$ was chosen as prescribable parameter. As $\mathrm{N}_{\mathrm{t}}$ increases, the temperature and concentration increase.

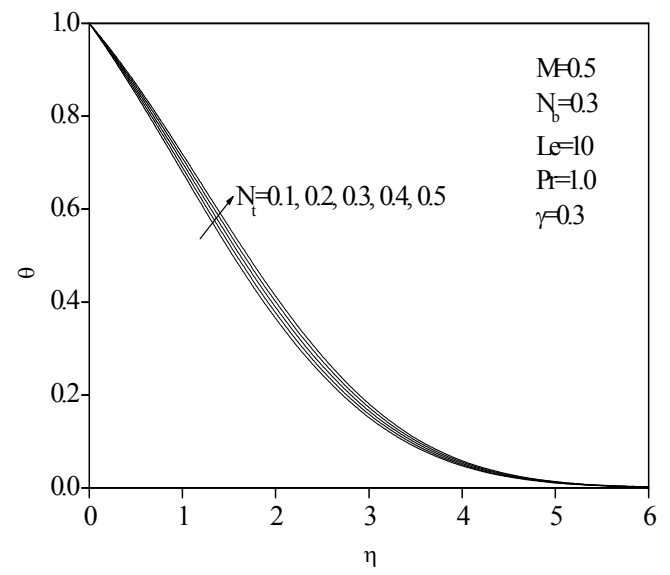

Figure 10: Effects of $\mathrm{N}_{\mathrm{t}}$ on temperature profiles

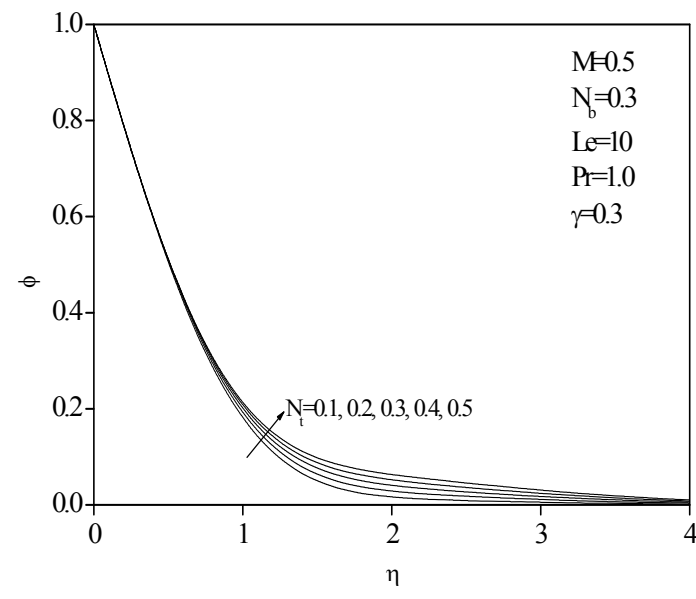

Figure 11: Effects of $\mathrm{N}_{t}$ on solid volume fraction profiles

Figures 12 and 13 show the variation of temperature and concentration as $\mathrm{N}_{\mathrm{b}}$ was chosen as prescribable parameter. As $\mathrm{N}_{\mathrm{b}}$ increases, the temperature increases whereas the concentration decreases.

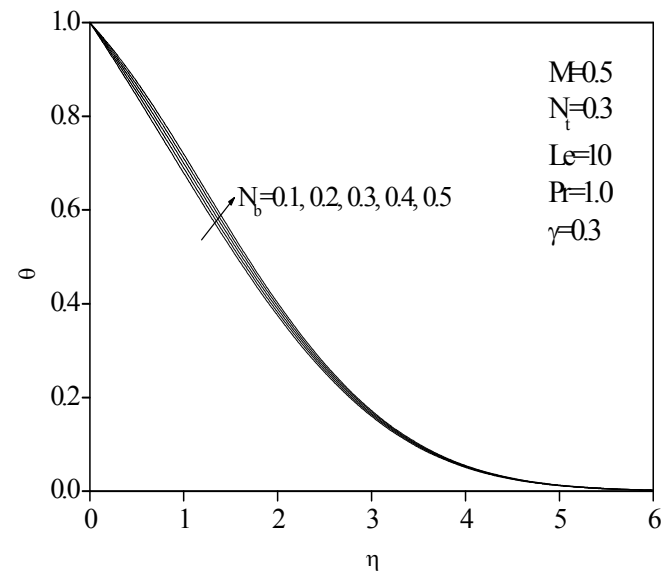

Figure 12. Effects of $\mathrm{N}_{\mathrm{b}}$ on temperature profiles

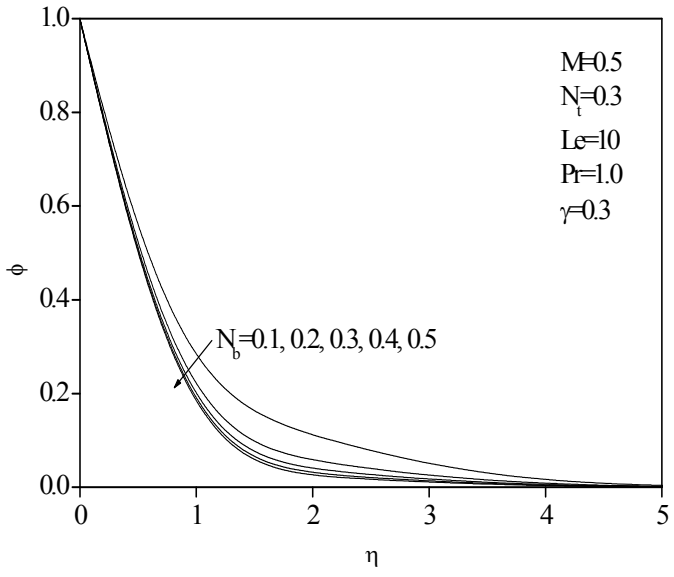

Figure 13: Effects of $\mathrm{N}_{\mathrm{b}}$ on solid volume fraction profiles

\section{Concluding Remarks}

In this work, we have studied the problem of the steady boundary layer flow of a warm, laminar nanoliquid over a melting surface moving parallel to a uniform free stream of the same material. The governing boundary layer equations are solved numerically using the fourth-order Runge-Kutta scheme along with the shooting method. The development of the Nusselt number and Sherwood number as well as the temperature, concentration and velocity distributions for various values of the velocity ratio, melting and nanofluid parameters has been discussed and illustrated in tabular forms and graphs. The results indicate that the melting phenomenon increases the heat transfer rate, (Nusselt number) at the solid/fluid interface. The effect of the 
nanofluid parameters on the temperature and concentration distributions as well as the friction factor and heat and mass transfer depends on the ratio of the velocity of the plate and the free stream fluid velocity.

\section{References}

Acrivos, A., Shah, M. J. and Peterson, E. E. (1960): Momentum and Heat Transfer in Laminar Boundary-Layer Flows of Non-Newtonian Fluids Past External Surfaces, AIChE Journal, Vol. 6, pp. 312-317. http://dx.doi.org/10.1002/aic.690060227

Buongiorno, J. and Hu, W. (2005): Nanofluid Coolants for Advanced Nuclear Power Plants, Paper no. 5705, Proceedings of ICAPP, Seoul.

Choi, S.U.S., Zhang, Z.G., Yu, W., Lockwood, F.E., and Grulke, E.A. (2001): Anomalous Thermal Conductivity Enhancement On Nanotube Suspension, Applied Physics Letters, Vol. 79, pp. 2252-2254. http://dx.doi.org/10.1063/1.1408272

Eastman, J. A., Choi, S.U.S. Li, S., Yu, W. and Thompson, L.J. (2001): Anomalously Increased Effective Thermal Conductivities Containing Copper Nanoparticles, Applied Physics Letters, Vol. 78, pp. 718-720. http://dx.doi.org/10.1063/1.1341218

Gorla, R.S.R. and Sidawi, I. (1994): Free Convection on a Vertical Stretching Surface with Suction and Blowing, Applied Scientific Research, Vol. 52, pp. 247-257. http://dx.doi.org/10.1007/BF00853952

Gorla, R.S.R., El-Kabeir, S.M.M., Rashad, A.M. (2011) Heat Transfer in the Boundary Layer on a Stretching Circular Cylinder in a Nanofluid," AIAA Journal of Thermophysics and Heat Transfer, Vol. 25, pp. 183-186. http://dx.doi.org/10.2514/1.51615

Gorla, R.S.R. and Chamkha, A. (2011): Natural Convective Boundary Layer Flow over a Horizontal Plate Embedded in a Porous Medium Saturated with a Nanofluid, Journal of Modern Physics, Vol. 2, pp. 62-71. http://dx.doi.org/10.4236/jmp.2011.22011

Howell, T. G., Jeng, D. R. and De Witt, K. J. (1997): Momentum and Heat Transfer on a Continuous Moving Surface in a Power Law Fluid," Int. J. Heat Mass Transfer, Vol. 40, pp. 1853-1861. http://dx.doi.org/10.1016/S0017-9310(96)00247-5

Huang, C.L. and Shih, Y.P. (1975): Perturbation Solution for Planar Solidification of a Saturated Liquid with Convection at the Wall, International Journal of Heat and Mass Transfer, Vol. 18, pp. 1481-1483. http://dx.doi.org/10.1016/0017-9310(75)90263-X

Kakac, S. and Pramuanjaroenkij, A. (2009): Review of Convective Heat Transfer Enhancement with Nanofluids," International Journal of Heat and Mass Transfer, Vol. 52, pp. 3187-3196. http://dx.doi.org/10.1016/j.ijheatmasstransfer.2009.02.006

Khanafer, K., Vafai, K. and Lightstone, M. (2003): Buoyancy-driven Heat Transfer Enhancement in a TwoDimensional Enclosure Utilizing Nanofluids, International Journal of Heat and Mass Transfer, Vol. 46, pp. 3639-3653. http://dx.doi.org/10.1016/S0017-9310(03)00156-X

Magyari, E. and Keller, B. (2009): Heat and Mass Transfer in the Boundary Layers on an Exponentially Stretching Continuous Surface,” J. Phys. D: Appl. Phys, Vol. 32, pp. 577-585. http://dx.doi.org/10.1088/0022$\underline{3727 / 32 / 5 / 012}$

Nield, D.A. and Kuznetsov, A.V. (2009): The Cheng-Minkowycz Problem for Natural Convective Boundary Layer Flow in a Porous Medium Saturated by a Nanofluid, International Journal of Heat and Mass Transfer, Vol. $\quad 52, \quad$ pp. 5792-5795. http://dx.doi.org/10.1016/j.ijheatmasstransfer.2009.07.024 Nield, D.A. and Kuznetsov, A.V. (2009): Thermal Instability in a Porous Medium Layer Saturated by a Nanofluid," International Journal of Heat and Mass Transfer, Vol. 52, pp. 5796-5801. http://dx.doi.org/10.1016/j.ijheatmasstransfer.2009.07.023

Patel, H. E., Das, S. K., Sundararajan, T., Sreekumaran, A., George B. and Pradeep, T. (2003): Thermal Conductivities of Naked and Monolayer Protected Metal Nanoparticle Based Nanofluids: Manifestation of Anomalous Enhancement and Chemical Effects, Applied Physics Letters, Vol. 83, pp. 2931-2933. http://dx.doi.org/10.1063/1.1602578

edroso, R.I. and Domoto, G.A. (1973): Perturbation Solutions for Spherical Solidification of Saturated Liquids, Journal of Heat Transfer, Vol. 95, pp. 42-46. http://dx.doi.org/10.1115/1.3450002 
Rao, J.H., Jeng, D. R. and De Witt, K. J. (199): Momentum and Heat Transfer in a Power-law Fluid with Arbitrary Injection/Suction at a Moving Wall, Int. J. Heat Mass Transfer, Vol. 42, pp. 2837-2847. http://dx.doi.org/10.1016/S0017-9310(98)00360-3

Sakiadis, B. C. (1961): Boundary-layer behavior on a continuous solid surface: II-The boundary layer on a continuous flat surface, AIChE Journal, Vol. 7, pp. 221-225. http://dx.doi.org/10.1002/aic.690070211

Crane, L. J.(1972): Flow Past a Stretching Plate, ZAMP, Vol. 21, pp. 645-647. http://dx.doi.org/10.1007/BF01587695

Schowalter, W. R., (1960): The Application of Boundary-Layer Theory to Power-Law Pseudoplastic Fluid, AIChE Journal, Vol. 6, pp. 24-28. http://dx.doi.org/10.1002/aic.690060105

Tiwari, R.K., and Das, M.K. (2007): Heat Transfer Augmentation in a Two-sided Lid-driven Differentially Heated Square Cavity Utilizing Nanofluids, International Journal of Heat and Mass Transfer, Vol. 50, pp. 20022018. http://dx.doi.org/10.1016/j.ijheatmasstransfer.2006.09.034

Tsou, F. K., Sparrow, E. M. and Goldstein, R. J. (1967): Flow and Heat Transfer In The Boundary Layer in the Continuous Moving Surfaces," Int. J. Heat Mass Transfer, Vol. 10, pp. 219-235. http://dx.doi.org/10.1016/0017-9310(67)90100-7

You, S. M., Kim, J. H. and Kim, K. H. (2003): Effects of Nanoparticles on Critical Heat Flux of Water in Pool Boiling Heat Transfer," Applied Physics Letters, Vol. 83, pp. 3374-3376. http://dx.doi.org/10.1063/1.1619206

Wang, C.Y. (1989): Free Convection on a Vertical Stretching Surface, ZAMM, Vol. 69, pp. 418-420. http://dx.doi.org/10.1002/zamm.19890691115

Wang, L. and Wei, X. (2009): Heat Conduction in Nanofluids, Chaos Solitons Fractals, Vol. 39, 2009, pp. 22112215. http://dx.doi.org/10.1016/j.chaos.2007.06.072 